\title{
Caracterización seminal de individuos ovinos criollos colombianos de pelo en el departamento de Sucre
}

\section{Characterization seminal of individuals hair sheep colombian creole in the department of Sucre}

\author{
CARRILLO-GONZÁLEZ DIEGO ${ }^{1}$ M.Sc, HERNÁNDEZ H. DARWIN ${ }^{*}$ Ph.D.
}

${ }^{1}$ Universidad de Sucre, Departamento de Zootecnia, Facultad de Ciencias Agropecuarias. Grupo de Investigación en Reproducción y Mejoramiento Genético Animal.

Campus Puerta Verde. Sincelejo. Colombia.

\section{Keywords:}

Reproduction, semen quality, zoogenetic resources, Colombia.

\begin{abstract}
The aim of this study was to characterize the semen of sheep individuals Colombian Creole hair on the farm at the University of Sucre. The study was carried out on the farm "El Perico" of the University of Sucre, 50 meters, warm dry climate and vegetation formation of tropical dry forest. four crossbred sheep adults, unrelated, which were collected four times at intervals of five days, using the technique of electroejaculation and seminal characteristics were evaluated macroscopic and microscopic were used. It was found that the predominant color is white matte $(66.7 \%)$ followed by bright white $(33.3 \%)(p<0.05)$, the seminal aspect is creamy $(60 \%)$, the average weekly volume is $1.41 \pm 0,11 \mathrm{ml}$, individual mobility is $74.09 \pm 2 \%$, gross motility is $3.77 \pm 0.12$ and the average sperm concentration of $711,89 \times 10^{6} \pm 133,86 \times 10^{6} \mathrm{esp} / \mathrm{ml}$. It was found that 76.64 $\pm 1.99 \%$ spermatozoa have normal morphology and $80.47 \pm 1.28 \%$ of the sperm had a functional plasma membrane. The seminal evaluation helped to show that the semen of crossbred sheep show similar to those reported in foreign breeds, although with higher volumes and higher percentage of membrane integrity features. It is concluded that the semen of Colombian creole hair sheep, has characteristics similar to other breeds quality, allowing it to be used in breeding programs.
\end{abstract}

\section{Resumen}

El objetivo de este trabajo fue caracterizar el semen de individuos ovinos criollos colombianos de pelo en la granja de la Universidad de Sucre. El estudio se llevó a cabo en la granja "EI Perico" de la Universidad de Sucre, a $50 \mathrm{msnm}$, clima cálido seco y con formación vegetal de bosque seco tropical. Se utilizaron cuatro ovinos criollos adultos, no emparentados, los cuales fueron colectados cuatro veces a intervalos de cinco días, mediante la técnica de electroeyaculación y se evaluaron las características seminales macroscópicas y microscópicas. Se encontró que el color predominante es el blanco mate $(66,7 \%)$ seguido del blanco brillante $(33,3 \%)(p<0.05)$, el aspecto seminal es cremoso $(60 \%)$, el volumen seminal promedio es $1,41 \pm 0,11 \mathrm{ml}$, la movilidad individual es $74,09 \pm 2 \%$, la movilidad masal es del $3,77 \pm 0,12$ y la concentración espermática promedio de $711,89 \times 10^{6} \pm 133,86 \times 10^{6} \mathrm{esp} /$ $\mathrm{ml}$. Se encontró que el $76,64 \pm 1,99 \%$ de los espermatozoides tenían una morfología normal y el $80,47 \pm 1,28 \%$ de los espermas presentaron una membrana plasmática funcional. La evaluación seminal permitió demostrar que el semen de los ovinos criollos, presentan características similares a las reportadas en razas foráneas, aunque con mayores volúmenes y mejor porcentaje de integridad de membrana. Se concluye que el semen de los ovinos criollos colombianos de pelo, presenta características de calidad similares a otras razas, lo que le permite ser usado en programas mejoramiento genético. 


\section{Introducción}

Los ovinos al igual que otros animales domésticos, no son originarios del continente Americano, sin embargo, presentan una amplia distribución geográfica, debido a su gran capacidad de adaptación a las diferentes condiciones de clima, vegetación y manejo (OCAMPO, 2014). Colombia cuenta con dos razas ovinas criollas, denominadas como de lana y de pelo. El primero de gran importancia económica para Boyacá, Cundinamarca, Nariño y Santander; la segunda se encuentra principalmente en la Guajira, Costa Atlántica, Llanos Orientales, Tolima, Valle del Cauca y Huila. Además se cuenta con una raza sintética llamada Mora Colombiana (CORPOICA, 2003).

Según el ICA (2016) el inventario ovino en Colombia es de 1.423.466 animales. Su producción ha estado tradicionalmente vinculada a una "economía de subsistencia" de bajo uso de insumos y relacionada con sistemas tradicionales y artesanales de producción, de tal forma que se concentra en pequeños rebaños, formados básicamente por sangre criolla en un 80 a $85 \%$ mestizos en un 10 a $15 \%$ y solo el $5 \%$ corresponde a los animales de razas puras o foráneas (GRAJALES y TOVÍO, 2010), generalmente traídos de Europa y otros países de America, entre las cuales se puede mencionar las razas Katahdin, Santa Inés, Pelibuey, Dorset, Dorper, Hampshire, etc., las cuales son utilizadas aparentemente por poseer mejores rendimientos productivos en comparación con los animales criollos, pero sin poseer la misma capacidad de adaptación a las condiciones del trópico (OCAMPO, 2014).

Los ovinos criollos presentan características adaptativas importantes al clima tropical, dentro de las cuales tenemos la tolerancia al calor, a los hectoparásitos y la capacidad de degradar pasturas de escaso valor nutricional, etc. Todo esto ha permitido la producción de estos individuos en condiciones medioambientales hostiles, facilitando actividades como el control biológico de malezas, el reciclaje de la biomasa y la obtención de abono orgánico y la producción de ingresos alternos en sistemas de producción destinados a la ganadería (GRAJALES y TOVÍO, 2010).

Los programas de mejoramiento genético en el ovino criollo de pelo son escasos, se realizan cruzamientos con razas importadas para aprovechar el vigor híbrido, o cruzamientos de tipo absorbente con razas europeas (OCAMPO, 2014).
En ninguno de los casos se utilizan biotecnologías reproductivas, como la inseminación artificial, la cual busca una ampliación en la difusión dirigida de las características superiores de machos mejoradores (BORRETO et al., 2002). La implementación y masificación de dicha biotecnología, requieren en primera instancia conocer aspectos básicos de la fisiología reproductiva y de la evaluación de la calidad seminal en la especie, todo con el fin de mejorar los índices productivos y reproductivos (GÓNGORA et al., 2010).

Diversas metodologías han sido propuestas para la evaluación de la capacidad reproductiva de los machos ovinos (carneros) las cuales incluyen la inspección físico-sanitaria del animal y la evaluación seminal. Esta última, es una herramienta utilizada principalmente para determinar la capacidad de fertilización del espermatozoide, y por ende predecir el potencial fértil de un reproductor. Además, permite la selección de eyaculados para procesos de refrigeración o de congelación y la clasificación de los machos para la utilización en programas de monta directa o para la inseminación artificial. Sin embargo, esta evaluación no se emplea de manera cotidiana en el proceso de selección de machos reproductores en la producción ovina.

La evaluación seminal estándar, incluye varios parámetros que se han agrupado en características macroscópicas y microscópicas. En las primeras se cuentan el aspecto, el color, el pH y el volumen; en la segunda la concentración, la movilidad, la vitalidad, la morfología, pruebas de integridad de las membranas plasmática y acrosomal, y las determinaciones de trayectorias y velocidades de movimiento (EBEL, 2012). Se ha asumido que estas medidas proveen información de la cantidad del eyaculado, de la calidad de la espermatogénesis y de la capacidad fertilizante del espermatozoide (BEDOYA et al., 2003; BEDOYA y PANTOJA, 2012; OLIVEIRA et al., 2010).

El ovino criollo de pelo colombiano, aparentemente presenta una producción espermática continua a través del año (NUÑEZ et al., 2014), sin embargo no se han definido los parámetros seminales normales de los machos criollos, ni se han implementado metodologías para su evaluación, que permitan establecer un criterio de selección a la hora de elegir un macho como animal reproductor, más allá de la simple evaluación de fenotipo. Por lo anterior, el objetivo de este trabajo 
fue caracterizar el semen de individuos ovinos criollos colombianos de pelo, en el Departamento de Sucre, para aplicar diferentes metodologías de evaluación seminal, que permitan mejorar el proceso de selección de machos mejoradores.

\section{Materiales y métodos}

Ubicación: La presente investigación se realizó en la granja experimental Los Pericos (9.210348 $\mathrm{N},-75.403194 \mathrm{~W}$ ), propiedad de la Universidad de Sucre, ubicada en el departamento de Sucre en el municipio de Sampués. A una altura sobre el nivel medio de mar de $160 \mathrm{~m}$, una temperatura promedio de $29^{\circ} \mathrm{C}$ y una precipitación anual de entre 1.000 y $1.200 \mathrm{~mm}$.

Animales y manejo: Se utilizaron 4 ovinos machos adultos criollos de pelo colombiano con edades entre los 1,5 y 4 años y pesos entre los 30 y $50 \mathrm{~kg}$. Todos los animales incluidos en el estudio se encontraban en condiciones saludables determinadas mediante un examen clínico general en el que se evaluó el peso vivo, las frecuencias y constantes cardíacas y respiratorias, la temperatura corporal, el estado de piel y pelaje, de mucosas y de nódulos linfáticos. También se realizó una inspección y palpación del aparato reproductor externo, la evaluación del comportamiento sexual y de la monta. Todos los animales fueron incluidos en el estudio si presentaban estructuras reproductivas normales y se encontraban sanos a la hora del estudio. Durante el estudio, los animales fueron mantenidos bajo un sistema semi-estabulado, con suministro agua y pastos del genero Brachiarias spp a voluntad.

Previa a la colecta de semen, a cada individuo se le efectuó una lavado prepucial con solución salina $0,9 \%$. La colecta seminal se realizó por el método de electroeyaculación, usando el equipo Electrojac6 $®$. Cada carnero fue colectado cuatro veces con intervalo de cinco días entre colecta, para un total de 16 eyaculados y cada eyaculado fue tomado como una repetición.

Mediciones: En cada eyaculado fueron evaluadas las variables macroscópicas de volumen, color y aspecto. El volumen fue determinado a través de la utilización de un tubo graduado de $15 \mathrm{ml}$ y el color y aspecto mediante la observación. Adicionalmente y bajo visión con el microscopio, se determinaron las variables de concentración espermática mediante la dilución 1:200 con agua destilada y el conteo en la cama de Neubauer
(MELLISHO, 2010). La movilidad masal e individual se determinó mediante la colocación de una gota de $30 \mu$ l en un portaobjetos y posteriormente se observó bajo visión con microscopio (Leica DM500) a un aumento de 100X y 400X respectivamente (MELLISHO, 2010). La morfología espermática se evaluó mediante la realización de extendidos de semen, teñidos con eosina-tinta china ( $50 \mathrm{~g} / \mathrm{L}$ de eosina) utilizando una

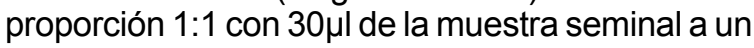
aumento de $1000 \mathrm{X}$, donde se contaron 200 células espermáticas (MELLISHO, 2010). Finalmente la evaluación de funcionalidad de membrana mediante la metodología de test hipo-osmótico (HOST) (OLIVEIRA et al., 2010), usando una dilución de semen en relación de 1:10 en solución de dextrosa al $5 \%$ (252 mOsms/L) e incubados en baño maría durante de 25 minutos a $25^{\circ} \mathrm{C}$, la evaluación se realizó por visión bajo microscopio en aumento de 400X.

Diseño experimental y análisis de datos: $\mathrm{EI}$ diseño experimental respondió a un modelo completamente al azar, con un sesgo de selección por conveniencia, los datos fueron analizados a través de un análisis de varianza, la comparación de las medias se realizó mediante el test de Tukey y se realizaron correlaciones entre las variables usando el programa estadístico StatPlus $₫$ :mac2009 ver 5.8.3.0. Otros resultados se presentan como porcentajes.

\section{Resultados y discusión}

Variables sensoriales: La evaluación del color del semen evidenció que el $66,7 \%$ de las muestras presentaron un color blanco mate, mientras que, el $33,3 \%$ restante, fue de color blanco brillante. Se encontraron diferencias significativas para este parámetro entre los animales evaluados $(p<0,05)$. El color y aspecto ideal del semen es blanco-lechoso o blanco-cremoso (CUETO et al., 2010; GARCÍA y URBANO, 2012), en el presente estudio, todos los eyaculados fueron de color blanco, los aspectos lechoso y cremoso tuvieron frecuencias de 26,7 y $60 \%$ respectivamente, aunque también se encontró un aspecto acuoso en el $13,3 \%$ de los eyaculados evaluados sin diferencias estadísticas entre los machos estudiados $(p=0,13)$. Desviaciones al color rojizo pueden indicar la presencia de sangre, así como los colores grises, marrones o amarillentos indican contaminación o infección, causales de desecho del eyaculado y posterior revisación del macho (CUETO et al., 2010). 
Variables macroscópicas: El volumen seminal promedio fue de $1,41 \pm 0,11 \mathrm{ml}$, sin presentar diferencias significativas $(p>0,05)$ entre eyaculados, lo anterior indica uniformidad para este parámetro en el semen de los ovinos criollos. Estos valores fueron superiores a los reportados por AL-SAMARRAE (2009) en las razas Karradi y Arrabi con volúmenes de $0,61 \pm 0,11 \mathrm{ml}$ y $0,59 \pm 0,03 \mathrm{ml}$ respectivamente, al igual que los mostrados por MOGHADDAM et al. (2012) en cruces de razas Iranies $(1,16 \pm 0,08 \mathrm{ml})$, por AGUIRRE et al. (2007), en la raza Pelibuey con un volumen promedio de $0,68 \pm 0,04 \mathrm{ml}$, mientras que, en las razas Españolas Menorquina y Galega se reportan valores de $1,06 \pm 0,11 \mathrm{ml}$ y $0,49 \pm 0,02 \mathrm{ml}$ respectivamente (VALLECILLO et al., 2011; FERNÁNDEZ et al., 2009). Una posible explicación para estos resultados es que la mayoría de los datos reportados son de razas en países templados, donde la reproducción de los ovinos obedece a ritmos estacionales, lo contrario ocurre en el ovino criollo colombiano, donde la reproducción es continua a lo lardo del año y por tanto el animal debe mantener la producción de semen.

Por otra parte JANETT et al. (2000) reportan volúmenes similares a los encontrados en este estudio en cruces de razas Suffolk, Merino y Awassi. VALLECILLO et al. (2011) presentan diferencias significativas entre eyaculados con un coeficiente de variación (CV) de 59,65\%, el CV de los datos aquí mostrados es de $31,71 \%$ y con $p>0,05$ lo que muestra menor variación en el volumen de eyaculado en los animales criollos. La variación en el volumen del eyaculado esta afectado por el periodo de descanso dado a los animales entre colectas (HAFEZ Y HAFEZ, 2005), los datos presentados indican que un tiempo de descanso de cinco días entre colectas es ideal para que el parámetro no varíe.

Variables microscópicas: En la Tabla 1 se muestran los resultados para las características microscópicas evaluadas en los eyaculados de los machos ovinos criollos. Se observó una movilidad masal promedio de $3,77 \pm 0,12^{++}$ ( $p<0,05$ entre eyaculados), este resultado fue superior a los reportados en la raza Menorquina $\left(3,58 \pm 0,02^{++}\right)$(VALLECILLO et al., 2011) y a otras razas del medio oriente (AL-SAMARRAE, 2009; MOGHADDAM et al., 2012), pero inferiores a los reportados en la raza Merino (ABDEL-RAHMAN et al., 2000), en la oveja Galega $\left(4,33 \pm 0,08^{++}\right)$ (FERNÁNDEZ et al., 2009).
Tabla 1. Características seminales microscópicas de ovinos criollos Colombianos de pelo en el departamento de Sucre.

\begin{tabular}{|c|c|c|c|c|c|}
\hline Variable & $\mathrm{NE}^{1}$ & Media & $\mathrm{EE}^{2}$ & Mín & Máx \\
\hline Volumen, ml & 16 & 1,41 & 0,11 & 0,8 & 1,8 \\
\hline Mov. Masal (0-5) & 16 & 3,77 & 0,12 & 3 & 4 \\
\hline Mov. Individual, \% & 16 & 74,09 & 2,00 & 65 & 90 \\
\hline $\begin{array}{l}\text { Concentración, } \\
\text { esp/ml }\end{array}$ & 16 & $711,89 \times 10^{6}$ & $133,86 \times 10^{6}$ & 70 & 1570 \\
\hline Morfología, \% & 16 & 76,64 & 1,99 & 60 & 85 \\
\hline HOST, \% & 16 & 80,47 & 1,28 & 75 & 88 \\
\hline
\end{tabular}

${ }^{1}$ Número de eyaculados, ${ }^{2}$ Error Estándar

Por otra parte, la movilidad individual obtenida fue de $74,09 \pm 2 \%$ (Tabla 1). Esta movilidad individual fue superior a los reportados en diversas razas (FERNÁNDEZ et al., 2009; AL-SAMARRAE, 2009; MOGHADDAM et al., 2012; VALLECILLO et al., 2011), pero inferiores a los reportados en la raza Merino (ABDEL-RAHMAN et al., 2000). VALLECILLO et al. (2011) encontró diferencias significativas en la movilidad individual entre eyaculados de ovinos colectados en diferentes años, notándose que a mayor edad del animal menor es la movilidad individual, en el presente estudio no se encontraron diferencias para esta característica $(p>0,05)$ a pesar de que los animales no tuvieron la misma edad. Otros factores que pueden influir en la movilidad individual se pueden clasificar como endógenos y exógenos, los primeros tenemos: la edad del animal donador y tiempo de almacenamiento de los espermatozoides en el epidídimo, tiempo de maduración espermática, reservas de energía (ATP) en el espermatozoide y finalmente integridad de la membrana; en los segundos se cuentan: la osmolaridad del plasma seminal, el $\mathrm{pH}$ y la temperatura (HAFEZ y HAFEZ, 2005). El análisis de los datos mostró una correlación de 0,80 $(p<0,05)$ entre las movilidades masal e individual, lo que indica que cuanto mayor es una mayor es la otra.

La concentración espermática encontrada fue en promedio de $711,89 \times 10^{6} \pm 133,86 \times 10^{6} \mathrm{esp} / \mathrm{ml}$, no se encontraron diferencias estadísticas significativas entre los eyaculados $(p>0,05)$, y se evidenció una alta variación $(\mathrm{CV}=77 \%)$ para esta característica (min: 70 y max: $1570 \times 10^{6} \mathrm{esp} / \mathrm{ml}$ ). En contraste, estudios realizados por AL-SAMARRAE (2009) en las razas Karradi y Arrabi en Iraq muestran concentraciones menores $(121,88$ y 120,5 $\mathrm{x} 10^{6} \mathrm{esp} / \mathrm{ml}$ respectivamente). Por otra parte, 
MOGHADDAM et al. (2012), en cruces de razas Iranies reportan concentraciones mayores

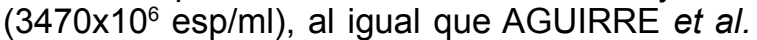
(2007) en la raza Pelibuey $\left(3880 \times 10^{6} \mathrm{esp} / \mathrm{ml}\right)$, VALLECILLO et al. (2011) en la raza Menorquina $\left(3900 \times 10^{6} \mathrm{esp} / \mathrm{ml}\right)$ y FERNÁNDEZ et al. (2009) en la Oveja Galela $\left(4197 \times 10^{6} \mathrm{esp} / \mathrm{ml}\right)$. Otros valores de concentración superior se encuentran en las razas Ansotana (QUINTÍN et al., 2005), la Manchega variedad negra (GONZÁLEZ et al., 1998), la Guirra (PUCHADES et al., 2004) y la Latxa (BELTRÁN DE HEREDIA et al., 2004).

En la prueba de morfología espermática se encontró un $76,64 \pm 1,99 \%$ de normalidad, en comparación con resultados reportados en diferentes razas tales como: Karradi $82,87 \%$, Arrabi $82 \%$ (AL-SAMARRAE, 2009) y cruces de razas Iranies 88,63\% (MOGHADDAM et al., 2012), se observó una tendencia de un menor porcentaje de normalidad de los espermatozoides en eyaculados de la raza Criolla Colombiana. Es necesario evaluar si el método de tinción utilizado (eosina-tinta china) en la presente investigación tiene repercusiones sobre este parámetro. De otro lado, es de resaltar que no se encontraron diferencias significativas $(p>0,05)$ entre los eyaculados. Algunos autores sugieren que existe una correlación positiva entre la morfología normal y la motilidad espermática (HAFEZ Y HAFEZ, 2005; MOGHADDAM et al., 2012) en el presente estudio esto no se observó. Sin embargo, HAFEZ y HAFEZ (2005) recalcan que aunque en todas las eyaculaciones hay algunos espermatozoides anormales, cuando se presenta el $20 \%$ o más de anormalidad, es necesario cuestionar la fertilidad del carnero.

La prueba de funcionalidad de membrana plasmática mediante la técnica HOST, evidenció que el porcentaje de espermatozoides que reaccionaron con el test fue del $80,47 \pm 1,28 \%$ con un valor mínimo de $75 \%$ y máximo de $88 \%$, sin diferencias significativas $(p>0,05)$ entre eyaculados. Resultados contrastantes son expuestos por OLIVEIRA et al. (2010) quien evaluando diferentes soluciones con osmolaridades entre 100 y $150 \mathrm{mOsmol} / \mathrm{L}$, reporta valores de $95,8 \%$ con citrato de sodio y de $76,9 \%$ con sacarosa. El porcentaje de HOST aca presentado es superior al reportado en la raza Merino $(74,1 \pm 7,6 \%)$ (ABDEL-RAHMAN et al., 2000). De igual forma, en semen criopreservado PELUFO et al. (2015) presenta un valor de $59,6 \pm 3,8 \%$ y BORRETO et al. (2002) de $35,5 \%$.
Estas diferencias encontradas, pueden deberse a la composición molecular de las soluciones empleadas y a efectos raciales de los animales, puesto que los tiempos de incubación según VÁSQUEZ et al. (2011) no generan diferencias entre los eyaculados ovinos.

BORRETO et al. (2002) correlaciona de forma positiva y significativa el porcentaje de HOST con la movilidad progresiva y el porcentaje de espermatozoides vivos, pero no, con el porcentaje de preñez. Los datos acá mostrados presentan una de correlación positiva entre las características morfología y movilidad individual con los porcentajes de HOST, aunque no fueron significativos. Lo anterior, explica que la presencia de cambios morfológicos en la cola de los espermatozoides (reacción positiva al HOST) no siempre es indicador la integridad funcional de la membrana acrosomal externa en la cabeza de los espermatozoides, la cual es imprescindible para la reacción acrosómica y la penetración del ovocito.

\section{Conclusiones}

Las variables evaluadas, demostraron que el semen de los individuos ovinos criollos del departamento de Sucre, presentan características similares a las reportadas en otras razas, con volúmenes del eyaculado y movilidades masal e individual superiores a otros reportes, pero, con concentración espermática variable. Aunque el porcentaje de normalidad espermática fue menor para los criollos, se evidenció que el semen presenta un mayor porcentaje de funcionalidad de membrana citoplasmática, lo que permite garantizar una posible eficiencia reproductiva. Las parámetros seminales evaluados no difieren significativamente entre eyaculados, esto asegura un estándar de calidad del mismo y hace que sean aptos para la utilización en programas de reproducción que permitan realizar procesos de mejoramiento genético usando biotecnologías reproductivas similares a la inseminación artificial. Adicionalmente la aplicación de protocolos de evaluación seminal, como parámetro para la selección de machos reproductores, permite ser más estricto en cuanto a la selección y/o descarte de animales posiblemente mejoradores.

Agradecimientos: Los autores agradecen a la Facultad de Ciencias Agropecuarias de la Universidad de Sucre por la financiación del proyecto. 


\section{Referencias}

ABDEL-RAHMAN, H.A.; EL-BELELY, M.S.; AL-QARAWI, A.A.; EL-MOUGY, S.A. 2000. The relationship between semen quality and mineral composition of semen in various ram breeds. Small Ruminant Research 38:45-49.

AGUIRRE, V.; ORIHUELA, A.; VÁZQUEZ, R. 2007. Effect of semen collection frequency on seasonal variation in sexual behaviour, testosterone, testicular size and semen characteristics of tropical hair rams (Ovis aries). Tropical Animal Health Production 39:271-277.

AL-SAMARRAE, S.H. 2009. Semen quality of arrabi and karradi iraqi rams. Diyala Agriculture Science J. 1:30-36.

BEDOYA, N.; VÁSQUEZ N.; RIVERA M.; CORREA, G.; TRUJILO, L. 2003. Evaluación de la integridad funcional de la membrana plasmática de espermatozoides bovinos mediante el test hiposmótico (HOST). Revista de la Facultad Nacional Agropecuaria de Medellin 56:1983-1997.

BELTRÁN DE HEREDIA, I.; GABIÑA, D. 2004. Producción y calidad de semen de los moruecos adultos de raza Latxa. Pequeños Rumiantes 5:8-12.

BORRETO, J.; GIBBONES, A.; BUNGE, M.; CUETO, M.; BIDINOST, F. 2002. Calidad seminal posdescogelamiento en relación con la eficiência reproductiva de la inseminación artificial laparoscópica en ovinos. Revista Medicina Veterinaria 83:185-188.

BEDOYA, P.; PANTOJA, C. 2012. Viabilidad espermática e integridade funcional del acrosoma en sêmen congelado de toros nacionales. Revista de Investigaciones Veterinarias del Perú 23:192-200.

CORPOICA - Corporación Colombiana de Investigación Agropecuaria. Ministerio de Agricultura y Desarrollo Rural. 2003. Situación de los recursos zoogenéticos en Colombia. Instituto colombiano agropecuario ICA. Bogotá, Colombia.

CUETO, M.; GIBBONS, A.; GARCIA, J.; WOLFF, M.; ARRIGO, J. 2010. Manual de obtención, procesamiento y conservación del semen ovino. Instituto Nacional de Tecnología Agropecuaria. Gobierno de Argentina. Buenos Aires, Argentina.

EBEL, F. 2012. Caracterización y pruebas de congelabilidad seminal en asnos Baudet du Poitou. Tesis de grado. Universidad Austral de Chile.

ICA, Instituto Colombiano Agropecuario. 2016. Censo Nacional Agropecuario 2016. Disponible en: http://www.ica.gov.co/getdoc/8232c0e5-be97-42bd-b07b-9cdbfb07fcac/Censos-2008.aspx. Consultada: 10-05-2016.

FERNÁNDEZ, M.; ADÁN, S.; LÓPEZ, C.; JUSTO, J.R.; RIVERO, J.C.; LAMA, J.J.; ROIS, D. 2009. Caracterización seminal de moruecos de la raza ovella Galega. Archivos de Zootecnia 58:533-536.

GARCÍA, J.L.; URBAJO, A. 2012. Manual de laboratorio para el análisis de semen. 1ra Edición. OmniaScience (Omnia Publisher SL).

Gongora, A.; MURILLO, R.; PEÑA, M.A.; RODRÍGUEZ, J.; RINCON-ARANGURI, M.; MORALES, R. 2010. Obtención y evaluación de las características seminales del Chiguiro (Hydrochoerus hydrochaeris). Memorias Conferencia Internacional de Medicina y Aprovechamiento de Fauna Silvestre Exótica 6:15-23.

GONZÁLEZ, M.E.; AGUADO, M.J.; PÉREZ-GÚZMAN, M.D.; MONTORO, V.; GIL, P.; GARDE, J. 1998. Técnicas reproductivas aplicadas a la conservación y fomento de la raza ovina Manchega, variedad negra. Arch Zootec. 47:329-334. 
GRAJALES, H.; TOVIO, N. 2010. Importancia de la oveja criolla colombiana como base genética en proyectos productivos. Universidad Nacional de Colombia sede Bogotá. Primer Congreso Internacional de Producción Ovina. Centro de Convenciones CAFAM - La Floresta. Bogotá, Colombia.

HAFEZ, E.S.E.; HAFEZ, B. 2005. Reproducción e inseminación artificial en animales. Séptima edición. Mc Graw Hill. Mexico.

JANETT; F.; HTISSY, D.; LISCHER, C.; H SSIG, I.M. THUN, R. 2001. Semen characteristics after vasectomy in the ram. Theriogenology 56:485-491.

MOGHADDAM, G.H.; POURSEIF, M.M.; RAFAT, S.A. 2012. Seasonal variation in semen quantity and quality traits of iranian crossbred rams. Journal Animal Science 45:67-75.

MELLISHO, E. 2010. Manual de laboratorio de reproducción animal. URL: http://tarwi.lamolina.edu. pe/ emellisho/reproduccionarchivos/practica\%204-eval-semen.pdf. Consultada: 5-06-2015.

NUÑES, F.; GARCIA, M.; BONAURA, M.; PRADEIRO, R.; GARCIA, M.; STORNELLI, M. 2014. Caracterización de semen obtenido mediante electroeyaculación en gatos domésticos. XV Jornadas de Divulgación Técnico-Científicas 2014 - II Jornada Latinoamericana Facultad de Ciencias Veterinarias Universidad Nacional de Rosario, Argentina.

OCAMPO, J.R. 2014. Caracterización genética de ovinos en Colombia por medio de marcadores microsatélites. Tesis de Maestría. Universidad de Antioquia. Colombia.

OLIVEIRA, L.; CORONA, M.; DAS NEVES, P. 2010. Diferentes soluções de teste hiposmótico para sêmen ovino. Revista Brasileira de Medicina Veterinaria 32:146-150

PELUFO, V.; LÓPEZ-ARMENGOL, M.F.; MALCOTTI, V.; VENTURINO, A.; AISEN, E.G. 2015. Effects of glycerol and sugar mixing temperature on the morphologic and functional integrity of cryopreserved ram sperm. Theriogenology 83:144-151.

PUCHADES, S.; MARCO-JIMÉNEZ, F.; RODRÍGUEZ M.; VICENTE, J.S. 2004. Adiestramiento y caracterización seminal de moruecos de la raza ovina Guirra. Archivos de Zootecnia 53:225-228.

QUINTÍN, F.J.; SEVILLA, E.; HERNÁNDEZ, M.; PASTOR, F.; VIJIL, E.; SOGUERO, J. 2005. Comportamiento sexual y rendimiento seminal en moruecos de raza Ansotana. XI Jornadas de Producción Animal AIDA. Zaragoza. España.

VALLECILLO, A.; MIRÓ-ARIAS, M.; ROCA, A.; PONS, A. 2011. Caracterización seminal de la oveja Menorquina. Archivos Zootecnia 60:349-352.

VÁSQUEZ, J.; FLORENTINI, E.; CAMARGO, L.; VALDIVIA, C. 2011. Test hipoosmótico en espermatozoides epididimarios en ovinos (Ovis aries). Spermova 1:119-120.

VIVAS, N.J. 2013. Diversidad genéticas de ovinos criollos colombianos. Tesis de Maestría. Universidad Nacional de Colombia. Colombia. 